\title{
A WBAN-based System for Health Monitoring at Home
}

\author{
Chris A. Otto, Emil Jovanov, and Aleksandar Milenkovic
}

\begin{abstract}
This paper describes a prototype system for continual health monitoring at home. The system consists of an unobtrusive wireless body area network (WBAN) and a home health server. The WBAN sensors monitor user's heart rate and locomotive activity and periodically upload time-stamped information to the home server. The home server may integrate this information into a local database for user's inspection or it may forward the information further to a medical server. The prototype may be used for ambulatory monitoring of patients undergoing cardiac rehabilitation or for monitoring of elderly at home by informal caregivers.
\end{abstract}

\section{INTRODUCTION}

$\mathrm{W}$ IRELESS body area networks are one of the mostsuitable technologies for building unobtrusive, scalable, and robust wearable health monitoring systems. A WBAN for health monitoring consists of multiple sensor nodes. Each node is typically capable of (i) sensing one or more physiological signals, (ii) processing these signals (e. g., filtering, feature extraction, and feature recognition), (iii) storing the processed data, and (iv) transmitting the data to other nodes and/or a WBAN server.

Continual advances in sensing technology, systems-on-achip (SoCs), wireless communication, and batteries promise further miniaturization, reduced sensor weight, increased processing power, and higher communication bandwidth. These advances combined with expected proliferation of wireless sensor network applications (e.g., environmental monitoring, home automation) will result in commoditization of wireless sensor network components. All these make it possible to design robust, cost-effective, and unobtrusive WBAN networks for personal health monitoring.

WBAN health monitoring systems may benefit a variety of users: from healthy outdoor enthusiasts who would like to track their fitness level during exercise, to users with impeding medical conditions or patients undergoing rehabilitation. Proliferation of such systems may prove crucial in promoting proactive approaches to healthcare. The importance of such systems is further underscored by current demographic, social, and economic trends (increased life expectancy, increased elderly population, increased costs in healthcare, and a lack of healthcare personnel) [1].

Chris A. Otto is with Lewis Innovative Technologies, Inc., Huntsville, AL (e-mail: chrisaotto@yahoo.com).

E. Jovanov is with the Department of Electrical and Computer Engineering, The University of Alabama in Huntsville, AL 35899 USA (email: jovanov@ece.uah.edu).

A. Milenkovic is with the Department of Electrical and Computer Engineering, The University of Alabama in Huntsville, AL 35899 USA (email: milenka@ece.uah.edu).
In this paper we focus on one particular deployment of WBAN-based health monitoring systems - health monitoring at home. In order to better understand design and operating issues of a WBAN system for health monitoring at home, we have built a prototype that consists of a motion sensor, a heart sensor, and a home health server application. The prototype has been tested by several users who wore the system from several hours to over a day. Our preliminary results indicate that the system prototype provides relatively robust operation without interfering with a user's normal activities.

The rest of the paper is organized as follows. Section 2 overviews the architecture and functionality of the proposed health monitoring system at home. Section 3 describes both hardware and software architecture of the prototype. Section 4 provides results of the system evaluation - detailing system performance, reliability, and wearability. Section 5 concludes the paper.

\section{SYSTEM ARCHITECTURE}

The architecture of the WBAN-based health monitoring system at home is multi-tiered - similar to the one described in [1] [2]. Tier 1 encompasses a set of tiny, smart, wireless sensors that are strategically placed on the user's body. These sensors sample, process, and store information about user's physiological signals. The WBAN sensors communicate directly to a WBAN gateway that may be plugged into either a home server or a wired or wireless network appliance (Fig. 1). The WBAN gateway provides time synchronization services (by transmitting periodic beacon messages) and forwards messages to a home server and/or a medical server. If a user moves out of the WBAN gateway range, the sensors automatically begin buffering data locally. When the user returns and the WBAN link is reestablished, the sensors automatically upload stored sensor and event data.

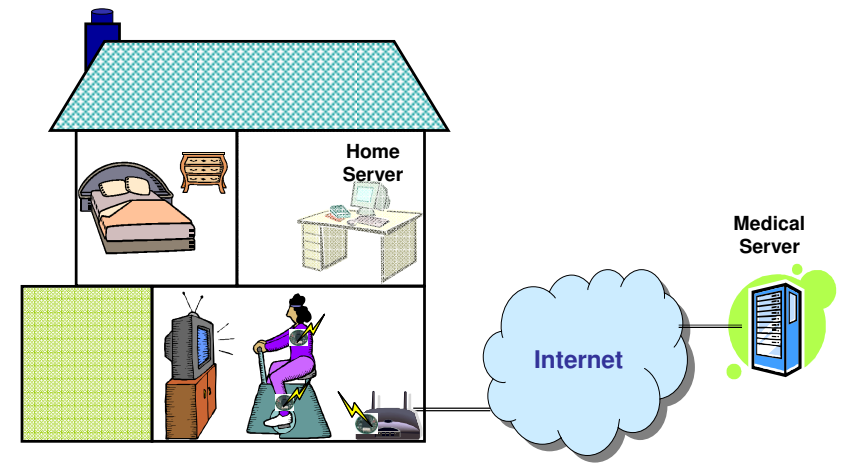

Fig. 1. System for health monitoring at home. 
In order to evaluate the overall system performance and specifically its wearability, reliability, robustness, and scalability we have built a system prototype. The main component of the WBAN-based system prototype and the data flow are illustrated in Fig. 2. The prototype features an activity (motion) sensor and a heart sensor. The motion sensors can be used to differentiate user activity states (e.g., sitting, walking, running, lying), or estimate intensity of his/her activity. Depending on the target application, the activity sensor can be attached to the user's belt, an ankle, a knee, or the trunk. More activity sensors can be deployed to achieve a more robust state differentiation and a better estimation of the user's activity. Once the deployment scenario is determined (exact position of the user's body), a user-specific sensor calibration may be needed to allow reporting of energy spent in calories.

The heart sensor monitors heart activity. One version of the heart sensor has a single-channel bio amplifier for threelead ECG. This sensor is capable of sending either raw ECG signal (signal is filtered) or R-peak events recognized by the on-sensor feature extraction software modules. The other version of the heart sensor interfaces a standard Polar belt and it can record each heart beat. As it does not require ECG electrodes (and thus increases the user's comfort), we used this heart sensor in our experiments. The user typically carries the heart sensor in his/her shirt pocket (close to the Polar belt). The WBAN gateway is implemented using a standard wireless platform. Finally, the home health server application runs on a personal computer.

The prototype may be used to monitor recovery and compliance of patients undergoing cardiac rehabilitation at home and who have been prescribed an exercise regiment. It can also be used in many other health monitoring applications with minimal or no modifications. In addition, the WBAN system is energy efficient, scalable, and its current implementation can accommodate more than a dozen sensor nodes as described in [4].

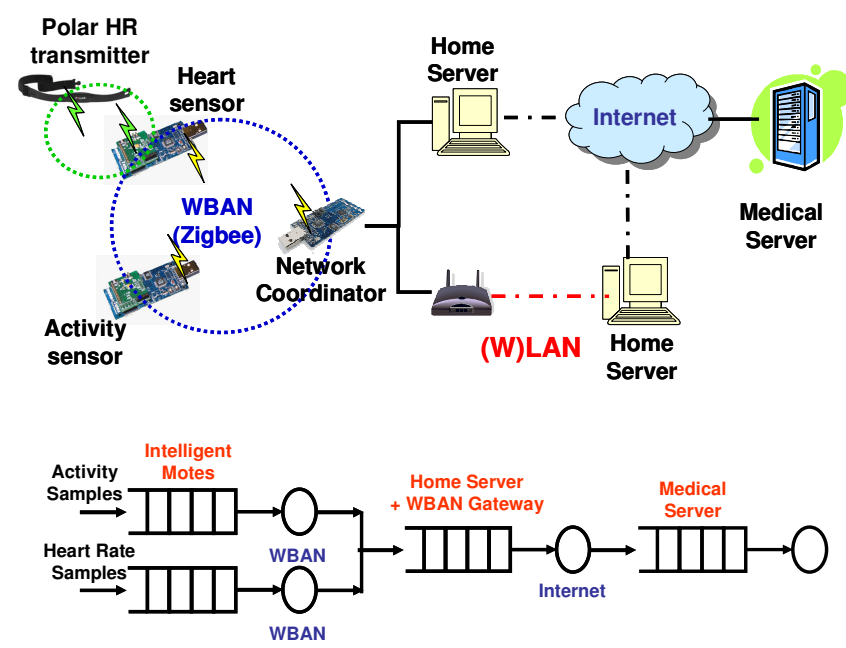

Fig. 2. WBAN prototype (top) and the data flow (bottom).

\section{HARDWARE AND SOFTWARE ARCHITECTURE}

\section{A. Hardware}

The activity WBAN sensor encompasses an intelligent daughter card with accelerometers and a connected Tmote sky wireless platform from Moteiv [3]. The daughter card samples acceleration in the $\mathrm{X}, \mathrm{Y}$, and $\mathrm{Z}$ axes; the raw signals are filtered and sent to the Tmote sky. The Tmote sky platform performs further processing (e.g., AEE computation, step detection, etc) and wirelessly transmits the processed data to the network coordinator.

The WBAN heart sensor encompasses a heart rate daughter card attached to a Tmote sky platform. The heart rate daughter card receives signals from a Polar WearLink wireless transmitter. The Tmote sky platform performs heart beat detection, time-stamping, and coordinates WBAN communication.

The WBAN network coordinator is implemented on a Tmote sky platform. It is responsible for WBAN gateway access and providing time synchronization services to the sensors in the WBAN. It can be connected directly to the home server or it can be connected to a Tmote connect appliance from Moteiv [3] and then accessed over the home network.

\section{B. Software}

The embedded sensor software is responsible for sampling and acquisition, real-time processing, event queuing, and WBAN communication. This software runs on the intelligent daughter cards and the Tmote sky platforms. Software running on sensor platforms and the network coordinator is developed using nesC language under TinyOS operating system. This implementation follows a general software architecture and WBAN communication protocol described in [1] [4].

The home health server is responsible for communicating with sensors in the WBAN, fusing sensor event messages as they arrive, generating per-user health monitoring session files, and providing visual and quantitative feedback to home users. In addition, it may include an agent responsible for data uploads to the medical server. The software runs on a home PC and was developed using Visual C\# and Microsoft .NET 2.0 framework. The .NET framework facilitates our handheld PDA version of the home health server as we have described in [1]. The home health server is capable of WBAN communications using either a direct connected network coordinator (USB) or a remote network coordinator connected via a Tmote connect. In the latter scenario, messages are transported over the local network using reliable TCP/IP sockets.

\section{Signal processing}

The WBAN prototype provides two parameters of user's activity: (a) activity-induced energy expenditure (AEE) and (b) RR-intervals for heart activity.

AEE. The AEE feature is calculated by processing 
accelerometer signals in real-time on WBAN sensor nodes. Employing embedded signal processing in this fashion extends battery life by reducing costly message transmissions (AEE updates every $\delta=15$ seconds versus $100 \mathrm{~Hz}$ raw sampling rate) and also promotes system scalability by minimizing processing and storage requirements from central server resources. Each axis is sampled at $100 \mathrm{~Hz}$ and prefiltered using simple low-pass averaging functions to remove frequencies above $20 \mathrm{~Hz}$. The resulting data stream is passed through a high-pass filter with an ultra low cutoff frequency $(0.01 \mathrm{~Hz})$ for the sole purpose of separating AC and DC signal components. The DC signal represents static acceleration due to gravity and is preserved for determining sensor orientation, discerning category of user activity, and step recognition. The AC signal component represents acceleration induced by user activity and is used in our AEE algorithm originally proposed by Bouten, et al [5]. It is described in Eq. 1, where $\mathrm{AC}\left(\mathrm{a}_{\mathrm{x}}\right), \operatorname{AC}\left(\mathrm{a}_{\mathrm{y}}\right)$, and $\mathrm{AC}\left(\mathrm{a}_{\mathrm{z}}\right)$ are $\mathrm{AC}$ components of accelerations on $\mathrm{x}, \mathrm{y}$, and $\mathrm{z}$ axes.

$$
A E E_{t}=\int_{t-\delta}^{t} \sqrt{A C\left(a_{x}\right)^{2}+A C\left(a_{y}\right)^{2}+A C\left(a_{z}\right)^{2}} \cdot d t \quad \text { Eq. } 1
$$

In our prototype, an activity event records $\mathrm{AEE}$ for $\delta=15$ seconds. A 4-byte AEE value is accompanied by a 4-byte time-stamp. It should be noted that the time-stamp may be redundant as the home health server may know the value of the time window $\delta$ used for AEE calculation, so just the first time-stamp in a session would suffice. However, in cases of a sensor malfunction or lost radio packets we might benefit from having time-stamps, thus achieving an increased level of confidence in our readings.

RR-intervals. The heart sensor detects and records Rpeak events. An R-peak event is described by two fields: an exact 4-byte timestamp of the R-peak and a 2-byte RRinterval (distance from the previous R-peak). The redundant format of the R-peak event allows full data recovery in all single occurrences of lost event messages.

\section{RESULTS}

\section{A. Event storage}

Buffering analysis for activity sensors. If an activity sensor node has not received a beacon message from the network coordinator, the current AEE sample with the corresponding time-stamp ( 8 bytes total) is buffered in a local buffer in on-chip RAM memory. Once the local buffer is full, the samples are stored in external flash memory. With $\delta=15$ seconds, we calculate the maximum out-ofrange operating time before event data is lost. The size of the local buffer is determined by the application requirements and for the activity sensor it is over $6 \mathrm{~KB}$. With 8 bytes produced every 15 seconds, we can store up to 192 minutes of time-stamped AEE samples [(6144 B $) / 32$ $\mathrm{B} / \mathrm{min}=192 \mathrm{~min}]$ in the local memory. If the external flash is employed, its capacity of $1 \mathrm{MB}$ would allow over 20 days of buffered time-stamped AEE samples.

Buffering analysis for heart sensors. One heart beat produces a 6-byte record. Assuming a typical heart rate range between $30 \mathrm{bpm}$ and $220 \mathrm{bpm}$, and similar memory budgets to the activity sensor, we can buffer from $4.65 \mathrm{~min}$ [6144 B / (6 B/b*220 b/min)] to 34.2 minutes of heart activity in the local RAM buffer, and from 13 hours [1 MB / $(6 \mathrm{~B} / \mathrm{b} * 220 \mathrm{~b} / \mathrm{min})]$ to 4 days if the external flash is used.

\section{B. WBAN Testing}

WBAN testing involved several users wearing the activity and the heart sensors for extended periods of time at home. In addition, we conducted testing in our laboratory with augmented user's activities. Below are results for two typical sessions.

Experiment \#1. Fig. 3 shows AEE and heart rate of a healthy user in his early 30s during 15 minutes of augmented activity in laboratory conditions. The experiment includes the following sequence of activities: 3 minutes of sitting, 1 minute of standing, 2 minutes of slow walking, 2 minutes of fast walking, 3 minutes of slow running, and 3 minutes of sitting. Increases in heart rate can be clearly seen at the beginning of periods with more activity (slow walking, fast walking, and running) simultaneous with increased AEE.

The user spent most of the time in a relatively close proximity of the network coordinator (distance less than 50 feet). Approximately $0.5 \%$ event messages were lost ( 10 out of 1,962 messages). All 10 of these lost packets were Rpeak event messages. Of these, only two were consecutive packets allowing full recovery of 9 out of 10 lost R-Peak events (by utilizing redundant RR-interval message fields).

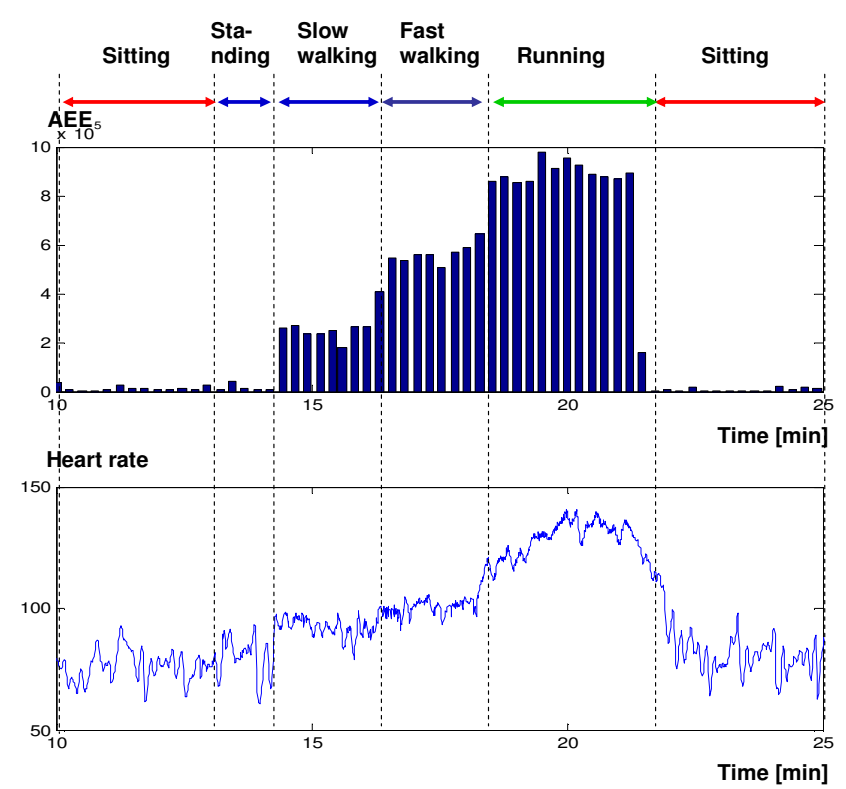

Fig. 3. AEE and heart rate collected on the home health server for 15 minutes with different types of activity. 


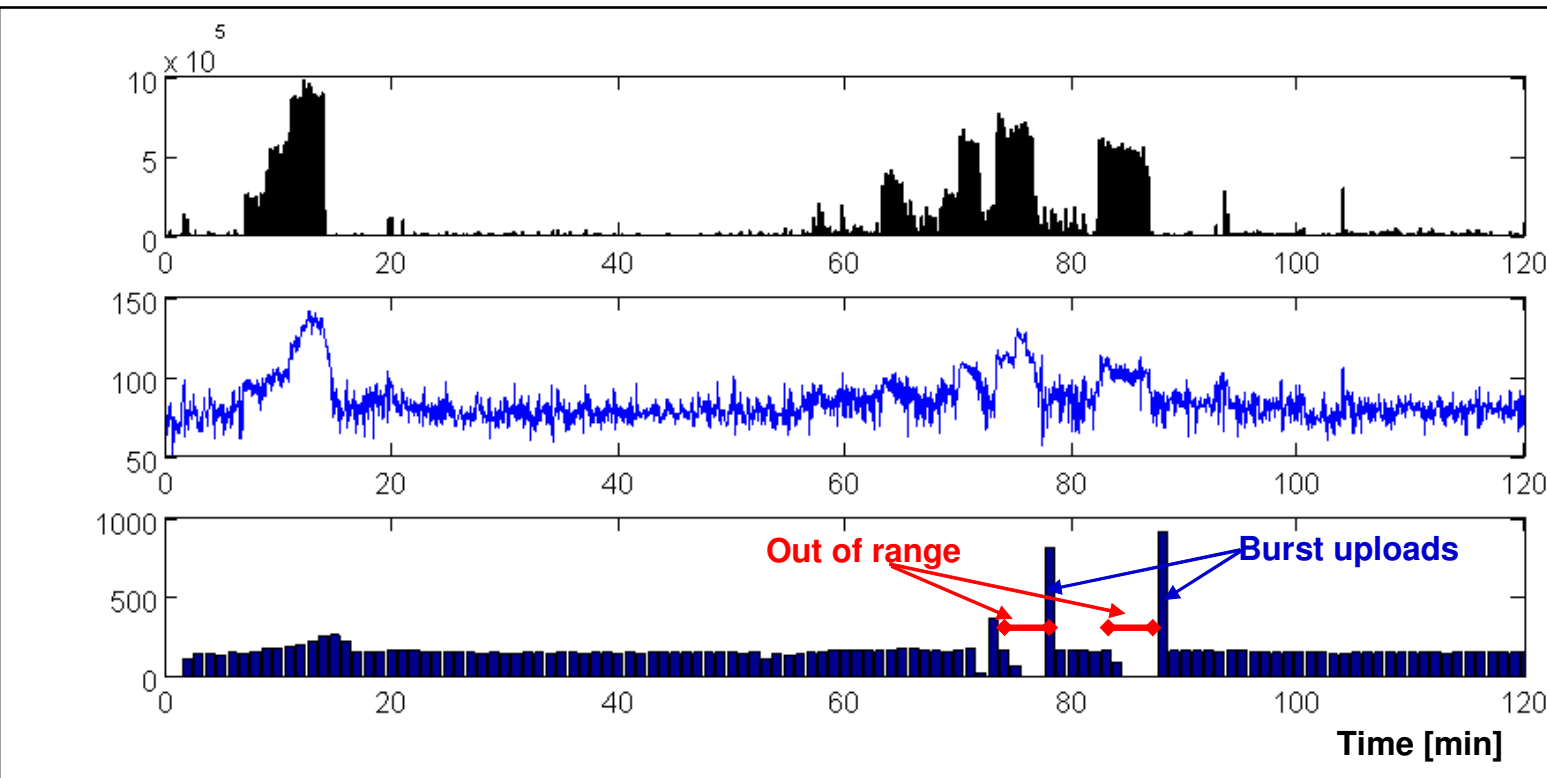

Fig. 4. AEE (top), heart rate (middle), the number of received packets by the network coordinator per minute (bottom) for a 2-hour session.

Experiment \#2. Fig. 4 shows AEE and heart rate for a 2 hour session. The user has been working in his office (sitting) with short periods of fast and slow walking. The bottom graph shows the number of received messages by the network coordinator per one minute. In this experiment the user walked out of the WBAN gateway range twice for relatively short periods of time (several minutes). Increases in the number of messages correspond to automatic uploads of buffered events once the user reenters the WBAN gateway range.

\section{CONCLUSION}

Based on testing, our WBAN-based prototype represents a viable system for health monitoring at home. Our experiences suggest potential for high user and patient compliance. The sensors are wireless and unobtrusive. In addition, system features such as event buffering and automatic uploads allow users to carry out normal activity.

By providing AEE and heart rate on-sensor, the system is already very useful for home health monitoring. Further research is required, however, in order to correlate activity and actual caloric consumption. Additionally, research is required to perform accurate step recognition and discern user activity states through software algorithms.

\section{ACKNOWLEDGMENT}

The authors thank Dr. Piet de Groen and Dr. Bruce Johnson from Mayo Clinic, Rochester, MN for many inspirational discussions and continual support.

\section{REFERENCES}

C. Otto, A. Milenkovic, C. Sanders, and E. Jovanov, "System Architecture of a Wireless Body Area Sensor Network for Ubiquitous Health Monitoring," Journal of Mobile Multimedia, vol. 1, 2006, pp. 307-326.

[2] E. Jovanov, A. Milenkovic, C. Otto, and P. C. d. Groen, "A Wireless Body Area Network of Intelligent Motion Sensors for Computer Assisted Physical Rehabilitation," Journal of NeuroEngineering and Rehabilitation, 2:6, vol. 2, March 1 2005.

[3] Moteiv, "Http://Www.Moteiv.Com," 2006.

[4] A. Milenkovic, C. Otto, and E. Jovanov, "Wireless Sensor Networks for Personal Health Monitoring: Issues and an Implementation," Computer Communications vol. in press, 2006.

[5] C. V. C. Bouten, K. T. M. Koekkoek, M. Verduin, R. Kodde, and J. D. Janssen, "A Triaxial Accelerometer and Portable Data Processing Unit for the Assessment of Daily Physical Activity," IEEE Transactions on Biomedical Engineering, vol. 44, 1997, pp. 136-147. 